\title{
Um Plugin para Aprendizagem de Lógica e Programação no Portugol Studio Baseado em Sistemas Adaptativos com Trilhas de Aprendizagem
}

\author{
Adson M. da S. Esteves, Aluízio Haendchen Filho, André L. A. Raabe \\ Universidade do Vale do Itajaí (UNIVALI) - Itajaí, SC - Brasil \\ \{shinadson, aluizio.h.filho\}@gmail.com, raabe@univali.br
}

\begin{abstract}
In Brazilian universities, the number of dropouts and evasion rates in computing courses are among the highest. Researches indicate that the lack of time for study, untrained teachers, and difficulties in understanding the activities are the main reasons that lead to this reality. Based on constructivism theories, this work aims to develop and evaluate an adaptive system to provide programming learning path options for the learner. An intelligent tutor helps the student on choosing trails and exercises according to their difficulties, characteristics and knowledge acquired from the system previous users.
\end{abstract}

Resumo. O número de desistências e as taxas de evasão nos cursos de computação estão entre as maiores nas universidades brasileiras. Pesquisas realizadas apontam que os principais motivos que levam a essa realidade são a falta de tempo para estudo, professores não capacitados, e dificuldades de entendimento das atividades. Com base em teorias do construtivismo, este trabalho tem por objetivo o desenvolvimento e avaliação de um sistema adaptativo para proporcionar ao aluno opções de escolha de trilhas de aprendizagem em programação. Um tutor inteligente auxilia o aluno nas escolhas de trilhas e exercícios de acordo com suas dificuldades, características e conhecimentos adquiridos de usuários anteriores do sistema.

\section{Introdução}

As disciplinas de Algoritmos e de Programação são os principais pilares dos cursos de computação. Essas disciplinas são consideradas difíceis por muitos alunos iniciantes, uma vez que exigem habilidades de abstração lógico-matemáticas, que eles não possuem em seu cotidiano [Raabe e da Silva, 2005]. As taxas de evasão podem variar de $22 \%$ a $32 \%$, sendo uma das maiores entre os cursos nas universidades brasileiras [Lobo, 2017].

Para reduzir essas taxas, Sistemas Tutores Inteligentes (STI), atualmente mais referidos como Sistemas Adaptativos, podem ser utilizados para facilitar e tornar o aprendizado menos complexo. Os STI são programas que incorporam técnicas de IA para o desenvolvimento de tutores que sabem o que ensinam, para quem ensinam e como ensinar [Nwana, 1990]. Entretanto, as soluções existentes ainda não contemplam as preferências e escolhas pessoais dos alunos enquanto disponibilizam atividades de programação [Crow 2018]. 
Visando aproximar as preferências dos alunos ao estilo de aprendizagem, foi selecionado o construtivismo como abordagem do STI. Ele se destaca por proporcionar o protagonismo do ensino ao aluno. Na abordagem construtivista, durante o processo de aprendizagem, o aluno pode decidir como aprender e agir de maneira proativa na construção do conhecimento [Bada 2015]. Ao aprender um novo conhecimento, o aluno avalia seu conhecimento prévio com a realidade.

Este trabalho tem como objetivo desenvolver um Sistema Adaptativo seguindo a abordagem construtivista para o ensino de programação. O sistema disponibiliza trilhas de aprendizagem adaptativas, e utiliza o Portugol Studio (PS) [Noschang et al, 2014] como plataforma de ensino-aprendizagem. O PS é uma IDE de código aberto voltada para iniciantes. Ela permite que versões modificadas sejam criadas, e também o desenvolvimento de plugins sem a necessidade de gerar novas versões.

\section{Problema de pesquisa}

A reprovação é uma das principais causas do cancelamento e evasão nos cursos de computação [Palmeira, 2014]. Para um melhor conhecimento das causas, é necessário descobrir os motivos que podem causar a reprovação dos alunos. Campos et al. (2011), apontam que a falta de preparo prévio em lógica de programação, e a abordagem pedagógica em sala de aula, são os fatores que mais influenciam nas reprovações.

Giraffa e da Costa Mora (2016) realizaram uma pesquisa com alunos que trancaram a disciplina de Ciência da Computação. Eles relataram que os principais motivos são: (i) a falta de tempo para estudo, pois muitos alunos têm jornadas de trabalho extra-classe; (ii) professores não tão bem capacitados para atenderem diversos alunos; (iii) dificuldades de entendimento das questões nas salas de aula e atividades. $\mathrm{O}$ sistema adaptativo facilitaria ao aluno estudar em momentos fora da aula, com práticas adaptadas para a sua capacidade.

Considerando estas dificuldades, faz-se as seguintes perguntas de pesquisa: (1) Um sistema adaptativo para o ensino de programação em uma IDE focada para iniciantes, facilita o aprendizado? (2) A liberdade de escolha de trilhas de aprendizado pelo aluno traz benefícios para a motivação dos alunos? As métricas para avaliar estas questões serão quantitativas, obtidas com a utilização de questionários durante o período de ensino-aprendizagem.

\section{Solução Proposta}

A solução proposta utiliza técnicas de IA e de STI para o desenvolvimento de um sistema adaptativo com trilhas de aprendizagem. A Figura 1 mostra a arquitetura genérica do sistema, representada em dois módulos: a Plataforma Web e o Módulo do Tutor.

$\mathrm{Na}$ plataforma web atuam dois agentes: (i) Classificador, que tem por responsabilidade classificar os alunos de acordo com suas características; e (ii) Recomendador, para recomendar o melhor exercício para o aluno com base em conhecimentos adquiridos de outros alunos.

O Módulo Tutor possui três agentes: Aluno, Rastreador e Pedagógico. Além disso, possui uma plataforma MIDAS [Haendchen Filho, 2017] responsável pela 
VIII Congresso Brasileiro de Informática na Educação (CBIE 2019)

Anais dos Workshops do VIII Congresso Brasileiro de Informática na Educação (WCBIE 2019)

execução do sistema multi-agente, pelo gerenciamento de comunicação entre os agentes e pelos mecanismos de acesso ao banco de dados.

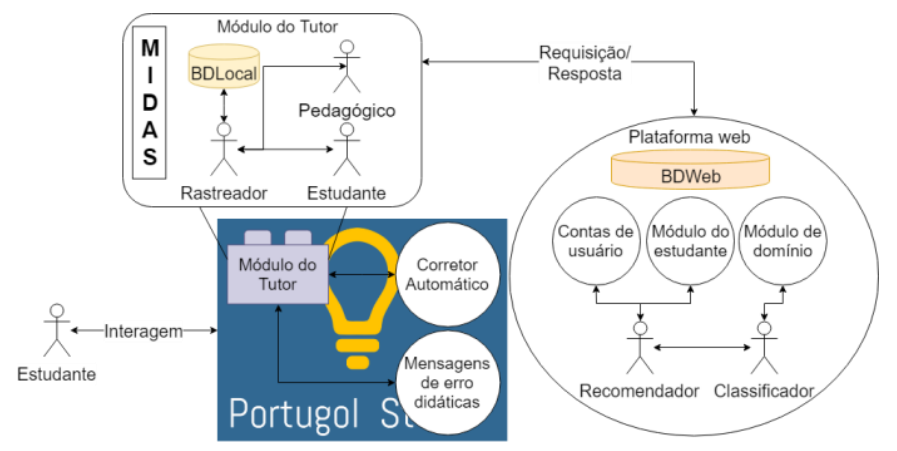

Figura 1. Arquitetura genérica do sistema proposto

Serão desenvolvidos os componentes da Plataforma Web e do Módulo do Tutor. O sistema utilizará um plugin para o PS desenvolvido por Pelz (2014) e Hodecker (2014). Esse plugin é um corretor de exercícios de programação que dá dicas aos usuários de erros cometidos e soluções para corrigi-los e finalizar o exercício. Além disso, serão utilizadas as mensagens de erro didáticas inerentes do PS para contribuir na ajuda ao aluno.

O sistema funcionará sem a necessidade de um professor fazendo manutenção ou atividades. Ele permitirá que qualquer usuário, em qualquer computador, possa utilizar o sistema sem restrições, desde que tenha o PS e o plugin instalados.

O sistema oferecerá as seguintes funcionalidades: (i) trilhas de aprendizagem com opções adaptativas, conduzidas por um tutor inteligente para que os alunos possam escolher os conhecimentos de seu interesse; (ii) dicas adaptadas de resolução e materiais de apoio durante a execução dos exercícios; e (iii) disponibilização de exercícios adaptados aos interesses pessoais do aluno.

\section{Justificativa}

Diversos tipos de pessoas que diferem em características como sexo, idade, nível de escolaridade, maneira de aprender e aptidão para a resolução de problemas, mostram interesse na área de computação e programação. Isto torna difícil a criação de um material único que atenda a todas as diferentes características [Oliveira et al, 2015]. Diversos trabalhos com sistemas tutores ou sistemas adaptativos [Crow 2018], foram desenvolvidos utilizando técnicas de agentes inteligentes de software ou sistemas multiagentes para tornar os ambientes mais adequados às características dos alunos.

No Laboratório de Inovação Tecnológica na Educação (LITE) da Universidade do Vale do Itajaí (UNIVALI), são pesquisadas novas formas de melhorar o aprendizado com a utilização de tecnologias. Foi no LITE que surgiu o Portugol Studio, hoje utilizado em mais de 7 universidades para facilitar o ensino de lógica e programação à iniciantes. A plataforma continua em constante evolução, e vários plugins têm sido desenvolvidos para torná-la mais atrativa e dinâmica para os usuários atuais e futuros. 
VIII Congresso Brasileiro de Informática na Educação (CBIE 2019)

Anais dos Workshops do VIII Congresso Brasileiro de Informática na Educação (WCBIE 2019)

\section{Metodologia}

Neste trabalho será aplicado o método hipotético-dedutivo. Serão coletados e analisados os dados relacionados com as questões de pesquisa para avaliar as hipóteses que serão levantadas. Quanto à abordagem, a pesquisa será quantitativa, pois após a utilização da solução em um ambiente controlado, será possível quantificar o quanto o seu uso auxiliou a aprendizagem do aluno de programação. Pela ótica da natureza, a pesquisa é aplicada, pois busca resolver problemas específicos com aplicações práticas.

Os seguintes procedimentos metodológicos estão previstos:

I. Preparação do corpus dos enunciados: aqui o objetivo será definir a sequência de enunciados do sistema, e classificá-la a partir das competências alcançadas.

II. Desenvolvimento: o objetivo nesta etapa é implementar no Portugol Studio um sistema tutor inteligente a partir dos enunciados definidos, utilizando técnicas de inteligência artificial fundamentadas na revisão da literatura.

III. Experimentos: esta atividade tem como objetivo realizar experimentos com alunos iniciantes em programação utilizando o sistema tutor desenvolvido.

IV. Análise dos Resultados: após a realização dos experimentos, o objetivo será analisar os resultados obtidos, comparando a proficiência adquirida pelos alunos que utilizaram a ferramenta com os alunos que não utilizaram.

\section{Resultados Esperados}

Espera-se, com este trabalho de pesquisa alcançar os seguintes resultados:

- Criar um plugin para a IDE Portugol Studio, tornando a ferramenta mais adaptativa com um tutor sugerindo materiais e exercícios mais adequados ao contexto pessoal e de aprendizado do usuário;

- Facilitar o aprendizado de iniciantes em programação, e desta forma, contribuir para diminuir a evasão nos cursos de computação;

- Construir uma base de dados de usuários iniciantes em programação, que possibilite o uso de técnicas de IA para gerar conhecimento;

- Possibilitar o uso do plugin fora da sala de aula, por iniciantes em programação, não necessariamente cursando computação.

- Gerar produção científica relevante, com a publicação de artigos em conferências e congressos nacionais e internacionais, e abrir novas possibilidades de uso das ferramentas em pesquisas;

\section{Contribuidores}

Neste projeto de Mestrado em Computação Aplicada, os seguintes professores atuam como orientador e co-orientador respectivamente:

O Dr. Aluízio Haendchen Filho, Doutor em Ciências-Informática pela Pontificia Universidade Católica do Rio de Janeiro (PUC-Rio). É Professor e Pesquisador do Programa de Mestrado em Computação Aplicada na Universidade do Vale do Itajaí (UNIVALI), onde participa dos grupos de pesquisa do Laboratório de Inteligência 
VIII Congresso Brasileiro de Informática na Educação (CBIE 2019)

Anais dos Workshops do VIII Congresso Brasileiro de Informática na Educação (WCBIE 2019)

Aplicada (LIA) e do Laboratório de Inovação Tecnológica na Educação (LITE). Ocupa o cargo de CEO na Smart Technology, uma Empresa de Base Tecnológica que desenvolve soluções inovadoras.

O Dr. André Luís Alice Raabe, Doutor em Informática na Educação pela PUCRS. Professor e pesquisador da UNIVALI, onde Coordena o Programa de PósGraduação em Computação e atua no Mestrado e Doutorado em Educação. Coordena o Laboratório de Inovação Tecnológica na Educação (LITE), onde o Portugol Studio é desenvolvido e o Grupo de Informática na Educação (GIE) da Univali.

\section{Referências}

Bada, S. O., \& Olusegun, S. (2015). Constructivism learning theory: A paradigm for teaching and learning. Journal of Research \& Method in Education, 5(6), 66-70.

Campos, A. M., Barbosa, L. S. and Fernandes, T. C. B. (2011). A new approach for teaching algorithms. Submitted to The 2011 International Conference on Frontiers in Education: Computer Science and Computer Engineering.

Crow, T., Luxton-Reilly, A., \& Wuensche, B. (2018). Intelligent tutoring systems for programming education. Proceedings of the 20th Australasian Computing Education Conference on - ACE '18. doi:10.1145/3160489.3160492

Giraffa, L. M., \& da costa Mora, M. (2013). Evasão na disciplina de algoritmo e programação: um estudo a partir dos fatores intervenientes na perspectiva do aluno. In Congresos CLABES.

Haendchen Filho, A. (2017) Sistemas Multiagentes na Internet: Uma arquitetura orientada a serviços para o desenvolvimento de agentes inteligentes de software na Internet, Rio de Janeiro: Novas Edições Acadêmicas.

Hodecker, A. Aprimoramento e avaliação do corretor de questões do Portugol Studio. 2014. 82 f. TCC (Graduação em Ciência da Computação) - Universidade do Vale do Itajaí, Itajaí, 2014.

Lobo, R. (2017) 'A Evasão No Ensino Superior Brasileiro - Novos Dados', Estadão, 07 October.

Noschang, L. F., Pelz, F., \& Raabe, A. (2014). Portugol studio: Uma ide para iniciantes em programaçao. Anais do CSBC/WEI, 535-545.

Nwana, H. S. (1990). Intelligent tutoring systems: an overview. Artificial Intelligence Review, 4(4), 251-277.

Oliveira, C. M., Pimentel, A., \& Krynski, E. M. (2015, October). Estudo sobre o sequenciamento inteligente e adaptativo de enunciados em programaçao de computadores. In Anais dos Workshops do Congresso Brasileiro de Informática na Educação (Vol. 4, No. 1, p. 1320).

Palmeira, L. B.; Santos, M. P. Evasão no bacharelado em Ciência da Computação da Universidade de Brasília: análise e mineração de dados. 2014. xvi, 127 f., il. Monografia (Bacharelado em Ciência da Computação) — Universidade de Brasília, Brasília, 2014.

Pelz, F. D. Um gerador de dicas para guiar novatos na aprendizagem de programação. 2014. 82 f. Dissertação (Mestrado em Computação Aplicada) - Universidade do Vale do Itajaí, Itajaí, 2014.

Raabe, A. L. A., Silva, J. M. C., (2005) "Um Ambiente para Atendimento as Dificuldades de Aprendizagem de Algoritmos". XXV Congresso da Sociedade Brasileira de Computação. São Leopoldo/RS. 